\title{
On the issue of damping external loads of mine racks
}

\author{
Kseniia DOMNINA ${ }^{1}$, Dariusz WIECEK ${ }^{2}$ and Valentin REPKO
}

Authors' affiliations and addresses:

${ }^{1}$ Votkinsk branch of Kalashnikov Izhevsk State Technical University, Department of Technical Mechanics, Shuvalova Street 1, 427430 Votkinsk, Udmurt Republic, Russia

e-mail: kseniya_domnina@bk.ru

${ }^{2}$ Faculty of Mechanical Engineering and Computer Science

University of Bielsko-Biala

Willowa 2 43-309 Bielsko-Biała, Poland

e-mail: wiecekd@ath.bielsko.pl

${ }^{3}$ Votkinsk branch of Kalashnikov Izhevsk State Technical University, Department of Technical Mechanics, Shuvalova Street 1, 427430 Votkinsk, Udmurt Republic, Russia

e-mail: repko@vfistu.ru

*Correspondence:

Kseniia Domnina, Votkinsk branch of

Kalashnikov Izhevsk State Technical University, Department of Technical Mechanics

427430, Shuvalova Street 1, Votkinsk, Russia

e-mail: kseniya_domnina@bk.ru

How to cite this article:

Domnina, K., Więcek, D. and Repko, V. (2020). On the issue of damping external loads of mine racks. Acta Montanistica Slovaca, Volume 25 (4), $532-541$

DOI:

https://doi.org/10.46544/AMS.v25i4.08

\begin{abstract}
The vulnerability of the main structural elements of rock liningsto external short-term and long-term loads is an important problem in the mining industry. The existing schemes are not designed for the simultaneous perception of compressive, shock and vibration influences. A way to solve this problem is to develop a new structural scheme of the racks, which are the linings' main bearing element. In the article, it is proposed to use multi-layer racks made as a rod covered with layers of plates. This will make it possible to use for damping external loads not only the pliability of the rack as a whole but also the frictional forces between its layers.The acting impact force will be decomposed into compressive and pressing forces. Due to the fact that the action of the external shock load is spent on moving and pressing the layers, its deformations are minimized compared to single-layer monolithic structures.For calculating the arising stresses, it is assumed that the rack plates are hinged at the edges, free along the length of the rack, and are under the joint action of compressive (stretching) forces and tangential forces evenly distributed on all sides of the plate.The article provides a rationale for the prospect of using such multi-layer racks and proposes an algorithm for calculating critical loads and stresses with the derivation of the damping coefficient. This coefficient is determined by the difference between the force of the shock wave and the critical load. The critical force, in turn, is calculated as the difference between the compressive force and the friction force between the layers, corrected for the part of the impact force, which causes stress close to critical in the dangerous middle section of the plate. By the value of the coefficient, it can be judged the effectiveness of the selected structural scheme of the rack.
\end{abstract}

\section{Keywords}

rack, multi-layer, damping, mine working, critical stress, stability 


\section{Introduction}

High rates of deepening and mining operations are typical for underground mining of ore deposits in the last 35-40 years. This is dictated by the rapid depletion of mineral reserves located near the earth's surface, as well as an increase in demand for a number of metals, especially those of strategic importance (Man-Chao et al., 2005; Nehring et al., 2018).

The most difficult and important problem in the development of such deep-lying deposits is the fight against changes in the stress state of the rock mass. These stresses tend to accumulate. And at a certain point, they can exceed the strength of rocks, which will begin to collapse with the release of significant energy ( Frankovský et al., 2020; Álvarez-Fernández et al., 2011; Gao et al., 2018).

Traditional structures of rock linings are often not designed for short-term shock and cyclic loads. This leads to the deformation of their elements under direct influence (Pivarčiová et al.,2019; Kanel et al., 2004; Pastor et al., 1990). Mine racks, which are used to hold and secure the walls and ceiling of mine workings, are the most vulnerable elements under shock loads. There is still no consensus among researchers on the most optimal design and material of the rack. To date, the number of patents for racks has exceeded 100. Such various options clearly indicate the lack of a generally accepted idea embodied in the optimal device. This caused an incessant design search that continues to this day.

\section{Stresses and deformations in soils under shock loads}

Shock loads cause wave processes in soils, which determine the stress state's level and the deformation associated with this level. Loads of this type cause thixotropic transformations of soils and rocks. As a result of such transformations, the ultimate strength and resistance of soils to external influences are reduced (Becker and Li, 2003; Zhang, X.W. et al., 2017; Pačaiová, 2017). Therefore, the study of processes occurring in soils under such loads is of great practical interest.

Shock loads excite more complex processes in soils compared to high-speed loads. Loads of this type can occur for the following reasons (Pecker, 2007; Yang et al., 2011):

a)explosions;

b)work of heavy impact machines (hammers, piling mechanisms);

c)impact development of solid rocks during their excavation, etc.

The movement of colliding bodies receives a finite, not a small, change upon impact in a very short period of time. As a result, very high pressures develop on their contacts. In this case, kinetic energy can be transferred to other types of energy.

The deformation of the soil that develops as a result of the impact is closely related to the stress state that occurs during this process. The vertical, i.e. compressive stress components, are particularly important. The time during which the soil is in a stressed state also affects. However, this effect is of secondary importance compared to stress (Dafalias and Popov, 1975).

An accurate calculation of dynamic phenomena occurring in rocks is associated with the following difficulties (Virieux et al., 2012; Kuric et al., 2017; Biały, 2013; Biały, 2014; Biały, 2015; Biały 2016):

- problems in integrating equations of motion and continuity;

- heterogeneity of rocks in terms of physical properties and structure;

- different reactions to the passage of plastic shock waves in soils.

The packing of the soil occurs when high stresses are applied to it, and a change in density under the action of pressure (medium stress) is observed. This change in density is usually characterized by volumetric deformation or volumetric compressibility $\Delta$ (Hendricks, 1980):

$$
\Delta=1-\frac{\rho_{0}}{\rho},
$$

where $\rho_{0}-$ undisturbed soil density.

This volumetric compressibility is an irreversible process, and tangential deformations lead to form change and permanent deformations in the soil.

At the same time, the shock wavefront can be considered as a surface on which the parameters characterizing the state and motion of the medium undergo a discontinuity. The basic laws of conservation of mass (2), the amount of motion (3) and energy (4) are fulfilled on the surface. The state of the medium before and after the rupture is characterized by the following parameters: shock wave velocity, medium density, medium flow velocity (Murthy, 2008): 


$$
\begin{aligned}
& \rho_{1} \cdot u_{1}=\rho_{2} \cdot u_{2} \\
& p_{1}+\rho_{1} \cdot u_{1}^{2}=p_{2}+\rho_{2} \cdot u_{2}^{2} \\
& \rho_{1} \cdot u_{1} \cdot\left(\frac{u_{1}^{2}}{2}+e_{1}\right)=\rho_{2} \cdot u_{2} \cdot\left(\frac{u_{2}^{2}}{2}+e_{2}\right),
\end{aligned}
$$

where $\rho_{1}, \rho_{2}-$ medium density, respectively, before and after rupture; $u_{1}, u_{2}-$ medium flow velocity, respectively, before and after rupture; $p_{1}, p_{2}-$ pressure, respectively, before and after rupture; $e_{1}, e_{2}-$ enthalpy, respectively, before and after rupture.

\section{Basic requirements for the operation of mine racks}

The demand for various types of mine racks is due to their wide range of applications. They are used as temporary and permanent lining elements in the construction of capital and preparatory mine workings, in sewage workings, etc. (Litvinskiy, 2019). The following restrictions exist for normal rack operation:

1) the rack is designed to perceive normal compressive force;

2) the appearance of eccentricity in the longitudinal force is one of the most dangerous states for the rack. This leads to bending and subsequent loss of stability;

3 ) the hinged thrust of both ends of the rack on the rock must be provided during installing. This is done to eliminate the load eccentricity;

4) the loss of longitudinal stability (according to Euler) is the critical limit state for the rack.

From the conditions of contact deformation-force interaction with the massif of rocks and soils, we note that the mine rack must additionally meet the following requirements:

- to ensure the maximum possible extensibility during installation to provide the required pliability margin;

- to have a smooth, without jerks and impacts pliability under the action of a load exceeding the level of working resistance;

- to switch to a hard mode of operation when the reserve of pliability is exhausted;

- to maintain longitudinal stability of compressed elements under load;

- not to deform (crumple or crush) rocks in contact with supports.

We assume that the most optimal design solution for the mine rack will be a rod consisting of plates with all the above conditions. This will make it possible to use for damping external loads not only the pliability of the rack as a whole but also the frictional forces between its layers. This is especially true when the critical stability load is exceeded for an already compressed rack (Saga et al., 2020; Vasko et al., 2020). The multi-layer structure of the rack will be an excellent damper in cases of shock and cyclic loads in the soil, including weak seismic loads. These loads act briefly, but the force of their impact is great due to the high velocity of the shock wave (Virieux et al., 2012). The structure of the rack will withstand a short load by damping its layers.

Such multi-layer structures require additional research.

\section{Materials and Methods}

The main provisions of the calculation of multi-layer mine racks are based on the theory of materials' discipline resistance. This concerns the part of determining stability within elastic deformations (Blatnicky et al., 2020). Let us list the key points.

1. After mining, the ceiling, walls and floor of the mine workings are limited by the zone with residual stresses. Residual stresses arise from drilling and explosion of rocks, movement of machines and mechanisms, etc.

2. A shock wave moves in the $x$-direction (fig. 1). The cause of the shock wave, as a rule, is the shift of the overlying layers of soil relative to each other. The shift occurs for the following reasons:

- undermining by groundwater flows;

- deformation of interlayers of light soils;

- surface shock-wave loads, etc.

The shock wave usually has a spherical shape and spreads from the point of explosion in all directions evenly, covering a huge space. Therefore, in the area of mine workings, this shock wave passes in places where there are residual stresses after cleaning the workings from rocks. The nature of the residual stresses is tension or compression. Residual tensile stresses do not last long because they expend their energy on the entire volume of the soil. Residual compressive stresses are the point. They increase at the points of the shock front (precisely at 
the moment of the shock wave passage). This is dangerous: the residual stresses with the shock wave cause deformation of layers of rocks and soil, which causes an impulse similar to a point explosion. And in this case, the velocity of the shock wave can increase to the maximum, i.e. to the speed of sound in this rock. Its energy will be directly proportional to the mass $\mathrm{m}$ of the stressed compressed rock:

$$
E=\frac{m v_{s}^{2}}{2}
$$

where $v_{s}-$ speed of sound in rock.

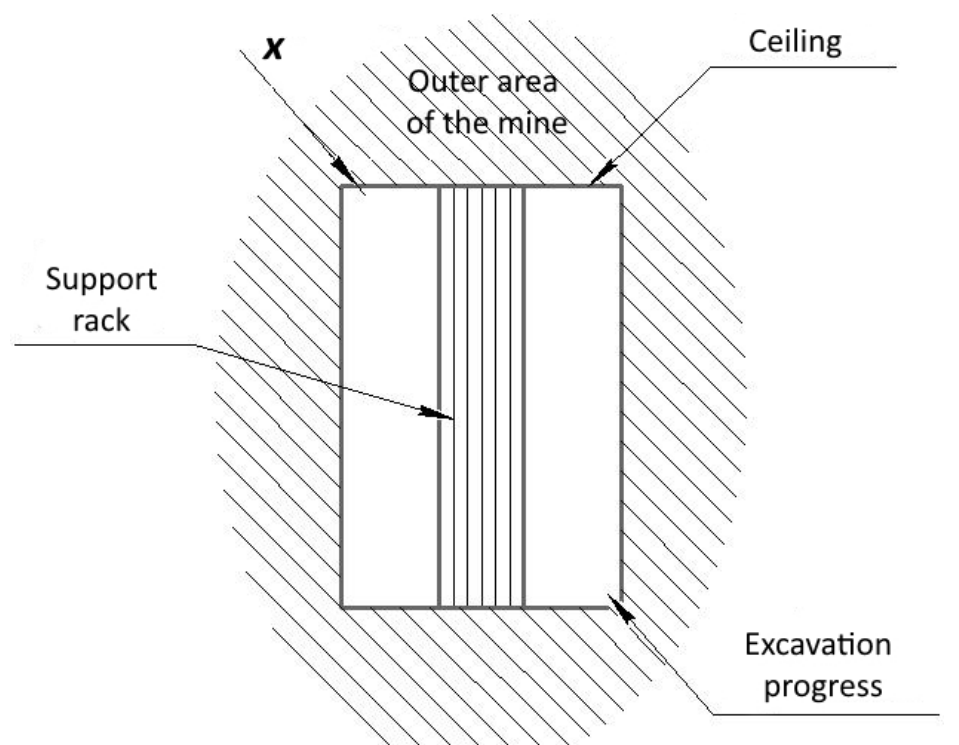

Fig. 1. Scheme of the position of the rack in the mine working

The impact on the rack will clearly exceed the critical load on the normative stability, and it will begin to bend. However, the impact velocity is so high that we can assume that the rack will remain in the zone of elastic deformations because the shock wave will quickly pass through it. The beginning of the bend of the rack is dangerous. It is the point when it is necessary to dampthe impact, which the layers will take on themselves, moving relative to each other and expending forces on this movement to overcome friction.

3. According to (Birger and Panovko, 1968), the total energy of the system $E$ is defined as the difference between the bending energy $U$ and the work of external forces $W$ :

$$
E=U-W
$$

Equality to zero of the expression (6) corresponds to the critical state. From this condition, critical combinations of edge loads can be found.

The forces of longitudinal deformation of the rack layers and the forces of friction between the layers are external forces.

a) forces of longitudinal deformation of the rack from impact

In fact, the rate of longitudinal deformation is very low. It can be measured in an experiment for a critical load but slightly less than the load of the rack exit from a stable position (Bozek and Turygin, 2014).

The shock wave is assumed to be similar to the sound wave in the rock in length and equal to the maximum impact force $P_{\max }$ in amplitude (fig. 2). By the duration of the impact $T_{0}$, we can find the work spent on the deformation of the rack. This work will reduce the work of the shock wave. 


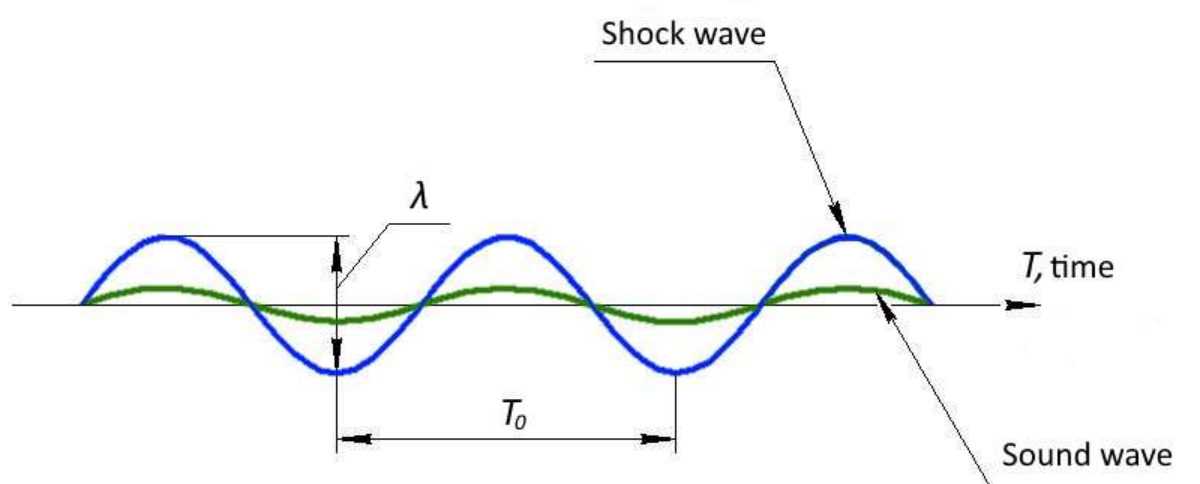

Fig. 2. Shock and sound waves in the circumferential zone

b) frictional forces

The rack begins to bend, moving the layers relative to each other (fig.3). A frictional force that prevents impact will occur at each joint

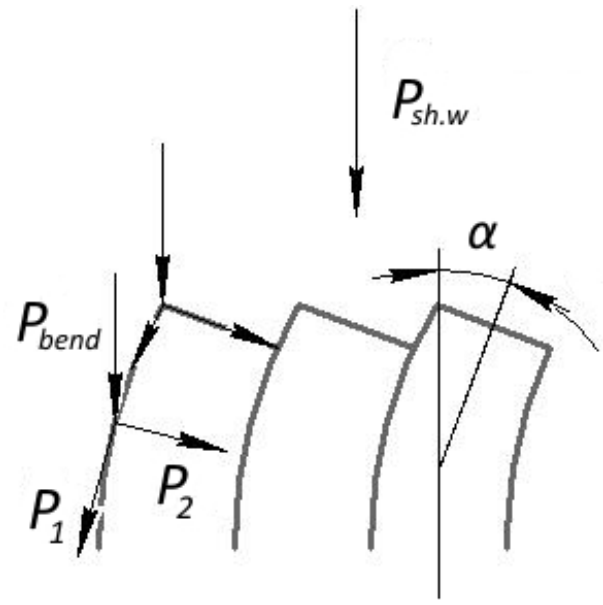

Fig. 3. Scheme of deformation of the rack layers relative to each other

Here $P_{1}$ is the force that continues the compression of the rack; $P_{2}$ is the force that presses the layers together:

$$
P_{2} \cdot k_{f r}=P_{d a m}
$$

where $k_{f r}$ - coefficient of friction between layers; $P_{d a m}$ - compensating damping force.

The equation (7) is valid for two layers. The angle $\alpha$ can be determined by the effect of the critical force on the rack's stability as a whole. The work of the $P_{\text {dam }}$ force set may eliminate the work of the shock wave force, taking into account the help of the forces' work on the compression of the rack layers. 2013):

4. The basic equation of the theory of bending of plates is the Sophie Germain-Lagrange equation (Kawano,

$$
\nabla^{2} \nabla^{2} w=\frac{q}{D}
$$

where $w$-deflection value; $q$ - distributed compressive load; $D$ - simplification, taken as:

$$
D=\frac{E h^{3}}{12\left(1-v^{2}\right)},
$$

where $E$ - Young's modulus; $v$ - Poisson's ratio; $h$ - plate thickness (here and hereafter is the constant). 
The solution of equation (8) must satisfy the boundary conditions reflecting the nature of the plate fixing and loading along the contour.

It is assumed that the rack plates are hinged at the edges, free along the length of the rack, and are under the joint action of compressive (stretching) forces and tangential forces evenly distributed on all sides of the plate (fig. 4) (Kienzler et al., 2004; Dodok et al., 2017).

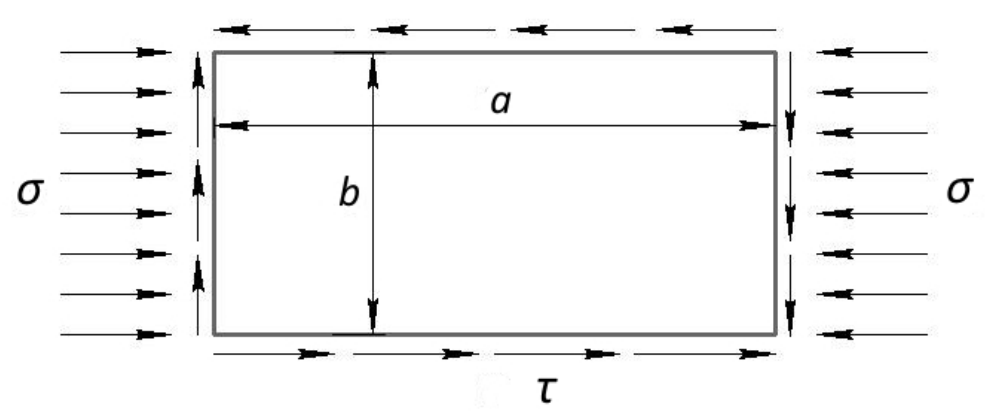

Fig. 4. Plate loading scheme

In this case, the critical stresses are:

$$
\begin{gathered}
\sigma_{c r}=K_{\sigma} \frac{\pi^{2} D}{b^{2} h} \\
\tau_{c r}=K_{\tau} \frac{\pi^{2} D}{b^{2} h}
\end{gathered}
$$

The values of the coefficients $K_{\sigma}$ and $K_{\tau}$ depending on the ratio of the sides of the plate $\lambda=\frac{a}{b}$ are given in Table 1. Here the minus sign in front of the coefficient $K_{\sigma}$ corresponds to tensile stresses.

Tab. 1. The values of the coefficients $K_{\sigma}$ and $K_{\tau}$ in the formulas (10)-(11)

\begin{tabular}{|c|c|c|c|c|c|}
\hline \multicolumn{2}{|c|}{$\lambda=1$} & \multicolumn{2}{c|}{$\lambda=1,6$} & \multicolumn{2}{c|}{$\lambda=3,2$} \\
\hline$K \sigma$ & $K \tau$ & $K \sigma$ & $K \tau$ & $K \sigma$ & $K \tau$ \\
\hline$-1,0$ & 10,57 & $-2,0$ & 8,46 & $-1,0$ & 7,45 \\
\hline 0 & 9,42 & 0 & 7,0 & 0 & 6,75 \\
\hline 1,0 & 8,15 & 2,0 & 5,31 & 1,7 & 5,4 \\
\hline 2,0 & 6,67 & 2,83 & 4,46 & 2,5 & 4,66 \\
\hline 3,0 & 4,72 & 3,6 & 2,95 & 3,0 & 4,14 \\
\hline 3,6 & 3,02 & 3,9 & 2,09 & 3,7 & 3,29 \\
\hline 4,0 & 0 & 4,2 & 0,06 & 4,017 & 2,19 \\
\hline
\end{tabular}

Normal stresses are more dangerous than tangents because they reach critical values faster.

We can calculate the values of the ultimate stress and critical load by determining the critical bending stress $\sigma_{c r}$ using the formula (10):

$$
\begin{gathered}
\sigma_{c r}=\frac{\sigma_{u l t}}{n} ; \\
\sigma_{c r}=\frac{P_{c r}}{A},
\end{gathered}
$$

where $\sigma_{u l t}-$ ultimate bending stress; $n$ - bending safety factor: $n>1 ; P_{c r}-$ critical bending load; $A-$ crosssectional area of the plate.

Based on the values of the critical loads and stresses, it is easy to carry out verification calculations and select the optimal material and dimensions of the cross-section of the rack. 


\section{Results and Discussion}

As a result, the rack can be calculated for deformation from the condition that in the critical mid-section, there are destructive bending stresses for each layer (Fig. 5).

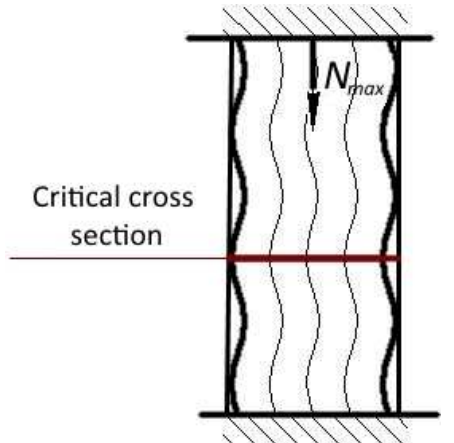

Fig. 5. Determination of the critical section of the rack during bending

This $N_{\max }$ will be the damping criterion. It is used to determine the range of protection against external shock loads of the working zone, shown in Figure 1.

We assume that the brevity of impact loads does not have time to bring the rack to destruction. However, due to the loss of stability, the rack will bend at a small angle $\alpha$. This angle will make it possible to decompose the acting force into two components, of which one continues to compress the rack, and the second is spent on overcoming the friction forces between the layers (Fig. 6). The friction force is found by the component of the external load directed perpendicular to the plate.

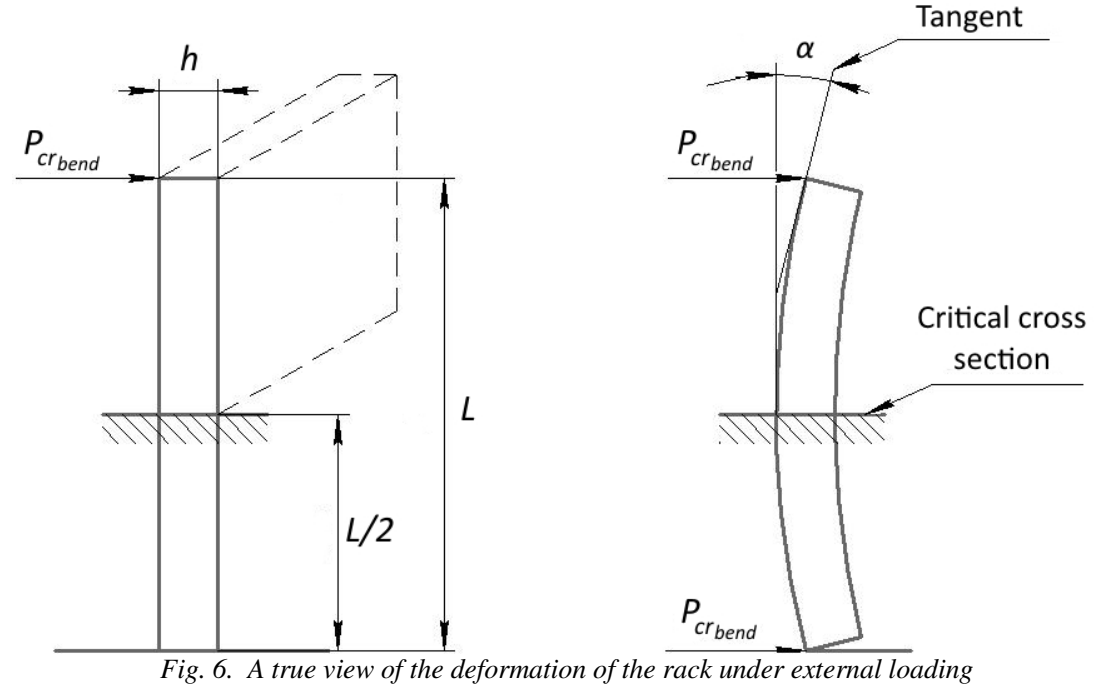

$P_{c r}$ is calculated from Figure 6 (Fig. 7).
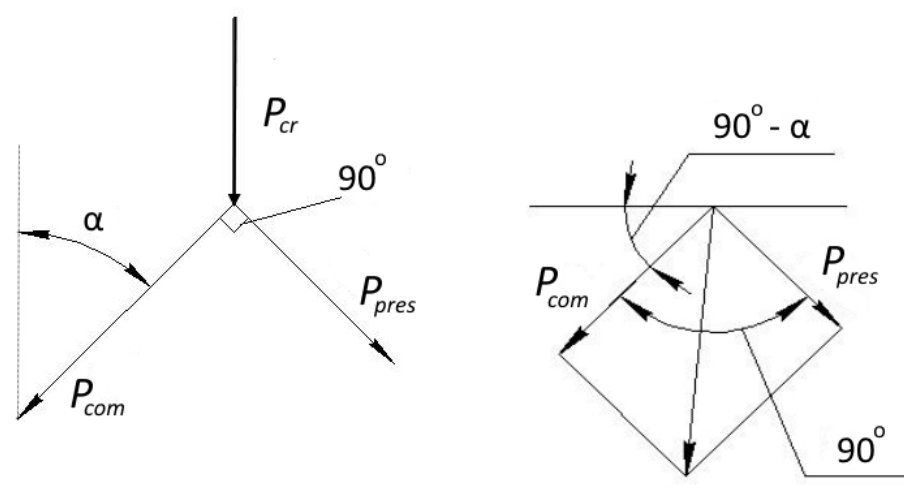

Fig. 7. Geometric scheme of the action of forces on a single plate 
Figures 3 and 7 show that $P_{\text {pres }}=P_{2}$, a $P_{\text {pres }} \cdot k_{f r}$ is the loss of compressive force $P_{\text {com }}$. In other words:

$$
P_{c r}=P_{c o m}-P_{p r e s} \cdot k_{f r}-P_{\text {com.loss }}
$$

where $P_{\text {com.loss }}-$ compression loss during the passage of a shock wave.

Obviously, the resulting force from $P_{c r}$ and $P_{\text {pres }}$ must be greater than each of these forces, for example, by $\Delta P$. The shock pulse $P_{c r}$ is found geometrically by $\Delta P$ from Figure 7 .

So it turns out that expression (14) will take on a new form (Fig. 8):

$$
P_{c r}=P_{c o m}-P_{\text {pres }} \cdot k_{f r}-P_{\text {com.loss }}+\Delta P
$$

where $\Delta P$ - the part of the impact force that, when the plate is being bent, causes stress in its middle section that is slightly less than $\sigma_{c r}$.

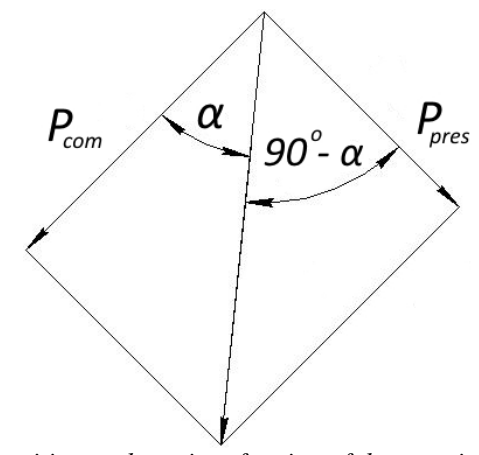

Fig. 8. Decomposition at the point of action of the pressing forces in the plates

This damping will give a simple result:

$$
P_{s h w}-P_{c r}=k_{d a m},
$$

where $P_{s h w}$ - shock wave force; $k_{d a m}$ - damping factor.

At $k_{d a m}>0$ - the rack will collapse, at $k_{\text {dam }}<0$ - the rack holds the impact and returns to its original state after removing the load. A monolithic rack has no layer damping, so it is weaker.

\section{Conclusion and Future Scope}

Taking into account the long overdue need for a change in scientific paradigms in the field of rock pressure management and the stability of underground workings, it is necessary to predict a transition from established dogmas to a more progressive solution of pressing problems (the form of mine working, new types and structures of linings, methods of protection and maintenance, etc.).

Mine racks are an element of the highest degree of responsibility in the construction of linings. Analysis of the existing requirements for the operation of racks revealed the need to revise their design and structure. Singlelayer monolithic structures are not competitive under conditions of simultaneous action of short-term and longterm compressive, shock and vibration loads. Therefore, it is proposed to use multi-layer racks made as a rod covered with layers of plates.

The effectiveness of the usage of multi-layer racks is justified by the well-known laws of resistance of materials. Due to the additional forces of interaction between the layers, it is possible to damp external shock loads in the zones of elastic deformation of the rack. The acting impact force will be decomposed into compressive and clamping forces, spending its energy on moving and pressing the layers, thereby minimizing the deformation of the multi-layer rack compared to single-layer monolithic structures.In addition to comparing the force of the shock wave and the resulting critical load in the strut, the derived damping coefficient allows planning an effective range of protection against external shock loads of the mine working area.

Of course, it is worthwhile to additionally think about the need to use devices, such as shock wave and vibration dampers.

Real experiments are required to develop an algorithm for calculating the structure of the rack (Delyová et al., 2021). It is also necessary to know the objective characteristics of the stress-strain state of the soil massif, namely: 
- values of vertical and horizontal stresses;

- stress concentration factor;

- cyclicity of loads;

- direction of the vector of action of the main stresses;

- elastic and total relative deformations of materials of racks and rocks on the contour of mine workings.

\section{References}

Álvarez-Fernández, M.I., González-Nicieza, C., Argüelles, A. (2011). Determination of the stress state in a rock mass subjected to excavation. Bull Eng Geol Environ. Vol. 70, 243-253. https://doi.org/10.1007/s10064010-0320-0.

Biały W.: New devices used in determining and assessing mechanical characteristics of coal.13 ${ }^{\text {th }}$ SGEM GeoConference on Science and Technologies In Geology, Exploration and Mining, SGEM2013 Conference Proceedings, June 16-22, 2013, Vol. 1, BULGARIA ISBN 978-954-91818-7-6/ISSN 13142704. pp. 547-554.

Biały W.: Coal cutting force measurment systems - (CCFM).14 ${ }^{\text {th }}$ SGEM GeoConference on Science and Technologies In Geology, Exploration and Mining, SGEM2014 Conference Proceedings, June 17-26, 2014, Vol. III, BULGARIA ISBN 978-619-7105-09-4/ISSN 1314-2704. pp. 91-98.

Biały W.: Monitoring of coal bed extraction as an effective tool for improving the production results of a mine.Management Systems in Production Engineering 3(19)/2015. ISSN 2299 - 0461. pp. 171-174. DOI: 10.12914/MSPE-11-03-2015

Biały W.: Determination of workloads in cutting head of longwall tumble heading machine.Management Systems in Production Engineering 1(21)/2016. ISSN 2299 - 0461. pp. 45-54. DOI: 10.12914/MSPE-0801-2016

Becker, T. and Li, T. (2003). Behavior of unsaturated soils subjected to cyclic loading. Proc. of Int. Workshop on Unsaturated Soils, Weimar, Germany: Springer, 347-364.

Birger, N.A., Panovko, Ya. G. (1968). Strength, stability, vibration. Handbook in three volumes. Moscow,Publishing house of Mechanical engineering, Vol. 3.

Blatnicky, M., Saga, M., Dizo, J. and Bruna, M. (2020). Application of Light Metal Alloy EN AW 6063 to Vehicle Frame Construction with an Innovated Steering Mechanism, Materials, Vol.13, Issue 4, Article No: 817 , DOI: 10.3390/ma13040817

Bozek, P. and Turygin, Y. (2014). Measurement of the operating parameters and numerical analysis of the mechanical subsystem. In Measurement Science Review. Vol. 14, No. 4 (2014), online, pp.198-203

Dafalias, Y.F. and Popov, E.P. (1975). A model of nonlinearly hardening materials for complex loading. Acta Mechanica, vol. 21, 173-192.

Delyová, I., Frankovský, P., Bocko, J., Trebuňa, P., Živčák, J., Schürger, B. and Janigová, S. (2021). Sizing and Topology Optimization of Trusses Using Genetic Algorithm. Materials, 14(4), 715.

Dodok, T., Cubonova, N., Cisar, M., et al. (2017). Utilization of strategies to generate and optimize machining sequences in CAD/CAM. Conference: 12th International Scientific Conference of Young Scientists on Sustainable, Modern and Safe Transport Location: High Tatras, Book Series: Procedia Engineering Volume: 192 Pp: 113-118

Frankovský, P., Brodnianská, Z., Bocko, J., Trebuňová, M., Kostka, J., Kicko, M. and Čarák, P. (2020). Application of holographic interferometry in the analysis of stress states in a crack root area. Applied optics, 59(13), D170-D178.

Gao, Y., Jiang, Y., Du, Y., Zhang, Q. and Xu, F. (2018). Vibration analysis of mountain tunnel lining built with forepoling method. Smart Structures and Systems, vol. 21, issue 5, 583-590.

Hendricks R.C. (1980) Reduced Volumetric Expansion and Isothermal Compressibility Factor Plots. In: Timmerhaus K.D., Snyder H.A. (eds) Advances in Cryogenic Engineering. Advances in Cryogenic Engineering, vol 35 A. Springer, Boston, MA. https://doi.org/10.1007/978-1-4613-9856-1_78.

Kanel, G.I., Fortov, V.E., Razorenov, S.V. (2004). Behavior of Brittle Materials under Shock-Wave Loading. In: Shock-Wave Phenomena and the Properties of Condensed Matter. High-Pressure Shock Compression of Condensed Matter. Springer, New York, NY, 111-178 https://doi.org/10.1007/978-1-4757-4282-4_4.

Kawano, A. (2013). A uniqueness theorem for the determination of sources in the Germain-Lagrange plate equation. Journal of Mathematical Analysis and Applications, 402, no. 1, 191-200.

Kienzler, R., Altenbach H., Ott I. (2004). Theories of Plates and Shells / Critical Review and New Applications. Springer. ISBN: 3-540-20997-2. DOI: 10.1007/978-3-540-39905-6.

Kuric, I., Cisar, M.; Tlach, V., et al.. (2019). Technical Diagnostics at the Department of Automation and Production Systems. Book Series: Advances in Intelligent Systems and Computing, Volume: 835, p. 474484, Published 
Litvinskiy, G.G. (2019). Mine pipe-concrete rack. Collection of scientific papers of "DonSTU", no. 17(60), 5-16.

Man-Chao, He, Xie, He-Ping, Peng, Su-Ping (2005). Study on rock mechanics in deep mining engineering. Chinese Journal of Rock Mechanics and Engineering, No. 16, 2804-2813.

Murthy, V. (2008). Geotechnical Engineering: Principles and Practices of Soil Mechanics and Foundation Engineering, ISBN-13: 978-0824708733.

Nehring, M., Knights, P F., Kizil, M.S., Hay, E. (2018). A comparison of strategic mine planning approaches for in-pit crushing and conveying, and truck/shovel systems. International Journal of Mining Science and Technology, Vol. 28, Is. 2, 205-214.

Pačaiová, H., Sinay, J., Turisová, R., Hajduová, Z. and Markulik, M. (2017). Measuring the qualitative factors on copper wire surface. Measurement, Vol 109, Pages 359-365.

Pastor, M., Zienkiewicz, O.C. and Chan, A.H.C. (1990). Generalized plasticity and modeling of soil behavior. International Journal for Numerical and Analytical Methods in Geomechanics, 151-190.

Pecker, A. (2007) Soil Behaviour under Cyclic Loading. In: Pecker A. (eds) Advanced Earthquake Engineering Analysis. CISM International Centre for Mechanical Sciences, vol 494. Springer, Vienna. https://doi.org/10.1007/978-3-211-74214-3_1.

Pivarčiová, E., Domnina, K., Ságová, Z. (2019). Design of the construction and research of vibrations and heat transfer of mine workings. Acta Montanistica Slovaca, Vol. 24, no. 1, 15-24.

Saga, M., Blatnicky, M., Vasko, M., Dizo, J., Kopas, P. and Gerlici, J. (2020). Experimental Determination of the Manson-Coffin Curves for an Original Unconventional Vehicle Frame, Materials, Vol.13, Issue 20, Article No: 4675, DOI: 10.3390/ma13204675

Vasko, M., Saga, M., Majko, J., Vasko, A. and Handrik M. (2020). Impact Toughness of FRTP Composites Produced by 3D Printing, Materials, Vol.13, Issue 24, Article No: 5654, DOI: 10.3390/ma13245654,

Virieux, J., Etienne, V., Cruz-Atienza, V., Brossier, R., Chaljub, E., Coutant, O., Garambois, S., Mercerat, D., Prieux, V. and Operto, S. (2012). Modelling Seismic Wave Propagation for Geophysical Imaging. Seismic Waves - Research and Analysis, Masaki Kanao, Chap.13. ISBN 978-953-307-944-8.

Zhang, X.W., Kong, L.W., Yang, A.W., Sayem, H.M. (2017). Thixotropic mechanism of clay: A microstructural investigation. Soils and Foundations, Vol.57, No.1, 23-35. https://doi.org/10.1016/j.sandf.2017.01.002.

Yang, C., Huang, M.S., Cui, Y.-J. (2011) Constitutive model of unsaturated structured soils under cyclic loading. Conference: 5th International Conference on Unsaturated Soils, September 6, 2010 - September 8, 2010, 987-992. DOI: 10.1201/b10526-152. 